\section{Risk indicators for dental caries using the International Caries Detection and Assessment System (ICDAS)}

Ismail AI, Sohn W, Tellez M, Willem JM, Betz J, Lepkowski J. Risk indicators for dental caries using the International Caries Detection and Assessment System (ICDAS). Community Dent Oral Epidemiol 2008; 36: 55-68. (C) 2007 The Authors. Journal compilation (C) 2007 Blackwell Munksgaard

Abstract - Background: While national surveys have found that AfricanAmericans have a higher prevalence and severity of dental caries than whiteAmericans, there are only a few descriptive studies of the prevalence and severity of dental caries in low-income urban African-Americans. Objectives: This study assessed the prevalence, severity and determinants of dental caries, using the International Caries Detection and Assessment System

(ICDAS). Methods: A representative sample of low-income families (a caregiver and a child aged 0-5 years) was selected from low-income census tracts in the city of Detroit, Michigan. Of the 12,655 randomly selected housing units, 10,695 were occupied and 9781 were successfully contacted (91.5\%). There were 1386 families with eligible children in the contacted households; and of those, 1021 were interviewed and examined at a permanent examination center organized for this study. This represents an overall response rate of $73.7 \%$. At the center, trained staff interviewed the main caregivers of the selected children, and trained and calibrated dentists examined the caregiver and her/his child. Data used in this study included information gathered from the social, behavioral and parenting questionnaires, the Block Food Frequency Questionnaire (total sugar intake), and data collected from community and census databases.

Results: Over $90 \%$ of the adults (ages 14-70 years, average 29.3) had at least one noncavitated carious lesion and $82.2 \%$ had at least one primary cavitated lesion. Negative binomial regression models found that the age of caregivers and the number of churches in neighborhoods were negatively associated with the number of noncavitated tooth surfaces. Cavitated tooth surfaces were positively associated with age, oral hygiene status, being worried about teeth, a recent visit to a dentist, and the number of grocery stores in the neighborhoods. However, the number of cavitated tooth surfaces was negatively associated with preventive dental visits, positive rating of oral health status and the number of dentists in a community. Conclusions: Dental caries, especially at the noncavitated stage, is highly prevalent in low-income African-American adults in Detroit. A

significant increase in the mean number of missing teeth was observed after the age of 34 years. This study found that different individual, social, and community risk indicators were associated with noncavitated versus cavitated tooth surfaces.
Amid I. Ismail ${ }^{1}$, Woosung Sohn ${ }^{1}$, Marisol Tellez ${ }^{2}$, Jenefer M. Willem ${ }^{1}$, James Betz ${ }^{1}$ and James Lepkowski ${ }^{3}$

${ }^{1}$ Department of Cariology, Restorative Sciences, and Endodontics, School of Dentistry, University of Michigan, Ann Arbor, MI, USA, ${ }^{2}$ Universidad El Bosque, Facultad De Odontologia, Bogota, Colombia, ${ }^{3}$ Institute for Social Research, University of Michigan, Ann Arbor, MI, USA

Key words: African-Americans; cavitated; dental caries; epidemiology; non-cavitated; social determinants

Amid I. Ismail BDS, MPH, MBA, DrPH, Department of Cariology, University of Michigan, School of Dentistry, Restorative Sciences and Endodontics, $1011 \mathrm{~N}$.

University Ave, D2361, Ann Arbor, MI 48109-1078, USA

Tel: 734-647-9190

Fax: 734-936-1597

e-mail: ismailai@umich.edu

Submitted 4 May 2006;

accepted 26 September 2006
Dental caries prevalence and severity has been declining in children and adolescents in the USA since the 1970s. The decline has been documented in different racial and socioeconomic population groups (1). There are, however, large population groups, such as minority and low-income individuals, 
who suffer from a disproportionately high severity of dental caries $(2,3)$ measured using traditional detection criteria and indices.

There are only a few studies that have evaluated the burden of dental caries in low-income AfricanAmerican adults. In one such study, low-income African-American adults aged 18-34 years living in New York had a mean of 8.8 decayed, missing, and filled teeth (DMFT). The mean almost doubled to 15.4 in adults aged 50-64 years (4). Missing tooth surfaces constituted about $43 \%$ of all the decayed, missing, and filled surfaces (DMFS) in the age group 18-34 years and increased to $72 \%$ in those who were 50-64 years old. Untreated dental caries constituted almost one-third of the total number of decayed and filled tooth surfaces (4).

National statistics on prevalence and severity of dental caries in children and adults have been collected by the National Health and Nutrition Examination Survey (NHANES) conducted by the National Center for Health Statistics. The NHANES selects a representative sample of the US noninstitutionalized population. The design, unfortunately, does not provide information on the oral health status of residents in small geographic areas such as states or cities. In 1999-2002, the NHANES found that about $85 \%$ of African-American adults had dental caries, with $41.3 \%$ having untreated decay. Among the white population, only $18.4 \%$ had untreated dental caries (5). African-American adults also had a higher number of missing teeth (mean $=6.9$ ) compared with white-Americans (mean $=4.3$ ) and with Mexican-Americans (mean $=4.3)$. White people had twice the number of filled teeth compared with African-Americans.

Current epidemiological data available on African-American adult caries is limited to descriptive statistics. Moreover, previous studies have collected data on dental caries using the Radike criteria (6), which group different stages of the caries process into one code (decayed) with no differentiation between noncavitated and cavitated lesions. This paper presents an analysis of data using the newly developed International Caries Detection and Assessment System (ICDAS) (7) that differentiates cavitated and noncavitated lesions.

New models on determinants of chronic diseases that investigate the role of community and individual risk factors on caries prevalence and severity have not yet been applied to the study of dental caries (8). Tellez et al. (9), using the same data analysed in this paper, found that the average total number of decayed tooth surfaces was negatively correlated with the number of churches in a community and positively correlated with the number of grocery stores. That analysis used the total number of decayed tooth surfaces (ICDAS codes 1-6); in this paper, we present findings of analyses of the epidemiology and risk indicators associated with noncavitated (ICDAS codes 1-2), cavitated (ICDAS codes 3-6), and filled tooth surfaces and missing teeth.

\section{Methods}

This paper presents findings of an analysis of the baseline data collected in 2002-2003 from a cohort of caregivers of young children in low-income families in Detroit, Michigan. The cohort of families is being followed up prospectively; data from the first follow-up phase are being prepared for analysis. This paper presents findings that will assist in developing the hypothesis for the subsequent longitudinal analyses.

\section{Sample selection}

A two-stage area probability sample was used to select a representative sample of low-income African-American children in low-income areas of the city of Detroit, Michigan. The 2000 Census data were used to identify 39 tracts with the largest proportions of households with African-American children from low-income families. A subset of the 325 Census tracts in Detroit was chosen as the study area, based on percentage of households below $200 \%$ of the poverty level, the percentage of households with African-Americans, and the percentage of households with children under age 6 years. Data on poverty level were collected during household screening to identify households meeting the inclusion criteria.

Power considerations and adjustment for anticipated attrition over the 4-year study period, indicated that a specified sample size of 1000 eligible children completing examinations would meet precision requirements for four different projects in the overall study.

A two-stage area probability sample of households and eligible children was selected from the study Census tracts. There were a total of 1526 blocks in these tracts. Census 2000 household counts were cumulated by block and a probability proportionate to size selection was used to choose 118 study blocks. For field data collection efficiency reasons, blocks with fewer than 100 households 
were linked to other blocks to form units of a minimum size of 100 households. The linking process resulted in 594 blocks linked together to form 118 study sample segments.

Within each selected segment, households were listed and chosen with probabilities inversely proportionate to size to obtain an equal chance of selection of households across the 39-tract study area. A team of specially trained community residents listed 14391 addresses in the 118 segments. The within-segment selection, with probability inversely proportionate to size, subsampled 12655 of the listed addresses.

Trained interviewers visited each sampled housing unit to screen its residents for eligibility. A total of 10695 sample housing units were occupied (84.5\% occupancy rate). Among the occupied housing units, 9781 were contacted and screened (91.5\% contact rate). There were 1386 (14.2\%) contacted and screened households with one or more eligible African-American children. One eligible child was selected as the index case in each household and the caregiver identified. A total of 1021 eligible children and their caregivers (see below) subsequently completed an interview and an examination in a centrally located study office. The combined screening and interviewing response rate was $73.7 \%$.

For the purpose of this analysis the sample segment was not a suitable unit for neighborhoodlevel analysis. To have a large-enough sample size to evaluate the impact of neighborhoods' risk indicators on dental caries, the responding households were grouped into neighborhood clusters by combining all segments from the same Census tracts (units used during sampling). However, Census tracts are administrative boundaries and do not necessarily represent objectively similar communities or neighborhood characteristics. Therefore, the original 39 Census tracts were further grouped into 21 neighborhoods based on three factors: (a) geographic proximity of tracts, (b) transportation patterns and street boundaries, and (c) location of neighborhood frames of reference, such as neighborhood associations. The neighborhood clusters were useful to gather communitybased information such as the number of dentists, grocery stores, and churches, and other relevant indicators.

The sample selection listing and screening phase was of 10-month duration and was conducted sequentially with data collection. The primary caregiver of the index child was recruited to participate in the study. The primary caregiver was defined as the person who has the decisionmaking authority about what the index child eats, how to take care of the index child's mouth and teeth, and when the index child visits the doctor or dentist, excluding those in a 'babysitting' capacity for the index child. The caregiver and index child were scheduled at the time of screening in the household to visit the project's Dental Assessment Center to be interviewed and examined. The design of the instruments for listing, methods of contacting and recruiting families, and all protocols were tested with focus groups that included members from the targeted community. The study protocol was approved annually by the Health Sciences Institutional Review Board of the University of Michigan.

\section{Examination and interviews}

Detection and classification of carious lesions

All subjects were examined by dentists trained specifically for study examinations. Dental examiner procedures were periodically calibrated to reduce inter-observer variability. Examinations were conducted using a portable dental chair and a halogen light. A compressor equipped with an air syringe, saliva ejector, and high speed suction was available for each examination. The examiners used plain mouth mirrors, air syringes, and World Health Organization (WHO) periodontal probes to check for surface discontinuity. Because of the length of the data collection phase (10 months), a team of four main dentists were supported by two back-up dentists [see Ismail et al. (10) for detail]. Two of the main dentists examined the families on weekdays and two other dentists worked on Saturdays. The dentists followed the ICDAS to measure the stages of the carious process. Both primary carious lesions and carious lesions adjacent to restorations and sealants (CARS) (what is commonly referred to as secondary or recurrent caries) were recorded.

The ICDAS system was developed in 2002 by a group of cariologists and epidemiologists after a review conducted for an international conference on clinical trials on dental caries identified wide variation among contemporary criteria systems (11). Table 1 presents a description of the basic ICDAS codes. The codes range from measurement of the first visible carious change in enamel (code 1) to extensive cavitation (code 6). Code 2 denotes more advanced noncavitated carious lesions where there is 'distinct visual change in enamel,' while 
Table 1. Diagnostic levels of dental caries measured using the International Caries Detection and Assessment System (ICDAS)

\section{Code Description}

0

Sound tooth surfaces

There should be no evidence of caries (either no or questionable change in enamel translucency after air-drying for $5 \mathrm{~s}$ ). Surfaces with developmental defects such as enamel hypoplasia; fluorosis; tooth wear (attrition, abrasion and erosion), and extrinsic or intrinsic stains are recorded as sound

1 First visual change in enamel

When seen wet there is no evidence of any change in color attributable to carious activity, but after air drying for $5 \mathrm{~s}$ a carious opacity is visible that is not consistent with the clinical appearance of sound enamel.

2 Distinct visual change in enamel

When viewed wet there is a carious opacity or discoloration that is not consistent with the clinical appearance of sound enamel (Note: the lesion is still visible when dry). This lesion may be seen directly when viewed from the buccal or lingual direction. In addition, when viewed from the occlusal direction, this opacity or discoloration may be seen as a shadow confined to enamel, seen through the marginal ridge

3 Initial breakdown in enamel due to caries with no visible dentin

Once dried for $5 \mathrm{~s}$ there is distinct loss of enamel integrity, viewed from the buccal or lingual direction. These lesions may also have a discolored dentine shadow beneath the marginal ridge

$4 \quad$ Noncavitated surface with underlying dark shadow from dentin

This lesion appears as a shadow of discolored dentin visible through an apparently intact marginal ridge, buccal, or lingual walls of enamel. The darkened area is an intrinsic shadow which may appear as grey, blue, or brown in color

5 Distinct cavity with visible dentin

Cavitation in opaque or discolored enamel with exposed dentine in the examiner's judgment

6

Extensive distinct cavity with visible dentin Obvious loss of tooth structure, the extensive cavity may be deep or wide and dentine is clearly visible on both the walls and at the base. The marginal ridge may or may not be present. An extensive cavity involves at least half of a tooth surface and possibly reaching the pulp

code 3 represents a condition where there is cavitation but only the enamel is visible in the walls and floor of the cavity. Code 4 describes a noncavitated stage with visible undermining of the enamel indicative of a carious process that extends into dentin. Codes 5 and 6 denote distinct and extensive cavitation with visible dentin, respectively. These six basic codes are the foundation upon which additional codes are added (first digit of a two-digit numbering system) to code the presence of sealants, restorations, and crowns, as well as the type of restorative material used. For example, code 4-0 is an amalgam restoration with no carious margins, while $4-2$ is a tooth surface with an amalgam restoration associated with a marginal visual carious change).

In addition to the detailed criteria, the examination protocol requires the assessment of pits and fissures separately from smooth tooth surfaces. On the buccal surfaces of the first, second, and third mandibular permanent molars, the buccal pits were scored separately from the smooth surfaces. Similarly, the lingual fissures on the first, second, and third maxillary molars, and the lingual pit on the maxillary central and lateral incisors, were scored separately from the smooth surfaces.

The ICDAS criteria integrate the experiences and criteria used in several previous studies (12-16). A description of the validity of the ICDAS criteria is reported by Ismail et al. (10). The intra-examiner reliability coefficients of the six examiners (two weekday, two weekend, and two back-up) in classifying teeth using each of the seven ICDAS codes (0-6) (Table 1) ranged between 0.65 and 0.91 (weighted kappa) (10). The inter-examiner weighted kappa coefficients ranged between 0.68 and 0.84 . The main examiners (weekday) examined 790 of the 1021 caregivers $(77.4 \%)$ and had inter- and intra-examiner weighted kappa coefficients that ranged between 0.83 and 0.91 .

\section{Assessment of oral hygiene status}

Before assessing the carious status of each tooth surface, the examiners applied a two-tone disclosing solution (PlaqueFinder; ProDentec, Batesville, AR, USA) to assess the efficiency of the oral hygiene procedures of the caregivers of the sampled children. The disclosing solution was developed based upon the research of Block et al. (17). PlaqueFind$\mathrm{er}^{\mathrm{TM}}$ contains two coloring agents: FDC Red \#2 and FDC Green \#3 (Fast Green). This mix of coloring dye stains mature plaque blue and new plaque red. The scoring method developed by PodShadely and Haley (18) was used to measure the location and age of the dental plaque on selected tooth surfaces. This method of assessing oral hygiene performance was found to be highly correlated (19) with the Plaque Index of Silness and Loe (20) and it was also 
found to be correlated with plaque weight. The intraexaminer reliability of this method of assessing oral hygiene performance was high $(r=0.95)$ (19).

To assess oral hygiene status, the examiners applied the disclosing solution on the buccal and lingual surfaces of six index teeth Fédération Dentaire Internationale [FDI] numbering system): $16,21,25,36,41,45$. After the teeth were rinsed, the examiner scored each tooth surface for the presence of plaque. Each tooth was divided into five segments: central one-third (gingival, mid-, and occlusal one-thirds), mesial one-third, and distal one-third. Old plaque, blue-stained plaque, was assigned a score of ' 2 ' and red-stained plaque (more recent plaque) was scored a ' 1 '. All participants brushed their teeth right before dental caries examination to thoroughly remove plaque and the disclosing agent. Examiners used air syringes to dry tooth surfaces examined based on the ICDAS protocol and used periodontal screening probes to remove any remaining plaque or debris to achieve maximum visualization.

Measurement of social, behavioral, and neighborhood-level indicators

Individual and neighborhood-level risk indicators were explored in bivariate analyses and final regression models. The dimensions and coding used for each variable were as follows: (a) Demographic variables: years of age (grouped as $1=14-24$ years; $2=25-34$ years; $3=35-44$ years; $4=45+$ years), education (grouped as $1=$ less than high school; 2 = high school graduate; $3=$ some college or higher $)$, sex $(1=$ male; $2=$ female $)$, family income $(1=$ less than $\$ 10000 ; 2=\$ 10000-$ 19 999; $3=\$ 20$ 000-29 999; $4 \geq 30$ 000), employment ( $1=$ yes; $2=$ no); (b) Access to care: type of insurance $(1=$ Medicaid; $2=$ private; $3=$ no insurance), last visit to dentist $(1=$ less than 2 years ago; $2=2-5$ years ago; $3=3-5$ years ago), reason for dental visit ( $1=$ preventive only; $2=$ treatment and prevention; $3=$ treatment only; $4=$ never been to dentist); and (c) Quality of life: self-perception of oral health $(1=$ Excellent/ Very Good; 2 = Good; 3 = Fair; 4 = Poor), worry about teeth $(1=$ a great deal; $2=$ somewhat; $3=$ a little; $4=$ not at all), pain caused by teeth $(1=$ a great deal; $2=$ some; $3=$ a little; $4=$ no pain). Oral hygiene status (oral hygiene performance index), total sugar intake in grams, number of churches, grocery stores, and dentists in neighborhoods were analysed as continuous variables.
Data about neighborhoods were obtained from two different sources. First, the online national yellow pages directory was used to obtain a list of addresses of dentists, churches, and grocery stores in each neighborhood in the zip code areas covering the study Census tract areas. Using a geocoding tool, 117 addresses of dentists, 478 addresses of churches, and 264 addresses of grocery stores were located in study neighborhoods. Secondly, the 2000 Census Summary Files 1 and 3 (SF1-SF3) provided indicators of characteristics on three main neighborhood dimensions: wealth, housing infrastructure, and social disadvantage:

- Percent of female headed households: A family with a female householder and no spouse of the householder present.

- Percent of households with public assistance income: Includes general assistance and Temporary Assistance to Needy Families, but not Supplemental Security Income.

- Percent of unemployed adults: All civilians 16 years old and over who were neither 'at work' nor 'with a job but not at work' during the reference week, were looking for work during the last 4 weeks, and were available to start a job.

- Earnings: The sum of wage or salary income and net income from self-employment (mean earnings rounded to the nearest whole dollar).

- Median household income: Income of the householder and all other individuals $\geq 15$ years old in the household, whether they are related to the householder or not.

- Residential mobility: Percent of persons over 5 years of age who lived at the same address for the past 5 years.

- Percent of households with no kitchen: A complete kitchen facility was defined as having all of the following: (i) a sink with piped water; (ii) a range, or cook top and oven; and (iii) a refrigerator.

- Percent of households with no plumbing: Complete plumbing facilities include: (i) hot and cold piped water, (ii) a flush toilet, and (iii) a bathtub or shower.

- Percent of people that use public transportation: Workers who usually used a bus or trolley bus, streetcar or trolley car, subway or elevated, railroad, ferryboat, or taxicab during the reference week as the principal mode of travel to get from home to work.

With such a large number of potential predictors, and observed high levels of correlation among some predictors, we used a factor analysis to 
reduce the number of 2000 Census variables. Eight indicators were reduced to three empirical factors. The first grouped the percentage of families that were female-headed (0.79 factor loading), percentage of households with public assistance income (0.82), the adult unemployment rate (0.71), and the percentage of people that use public transportation (0.36) into a 'Social Disadvantage' index. The second factor grouped the no plumbing (0.80) and no kitchen (0.82) variables into a 'Housing Infrastructure' score. The third grouped the median household income (0.83) and the earnings variables (0.82) into a 'Wealth' score. The three factor scores had Cronbach alpha coefficients greater than 0.70. The factor loadings for each factor were used to compute scores for each cluster for social disadvantage, housing infrastructure deficiency, and wealth. The resulting three factor scores were also combined to create a neighborhood socioeconomic disadvantage score. Standardized scores representing the number of standard deviation units of the value from the mean were computed. Higher social disadvantage scores denoted greater degrees of privilege in the neighborhood. All other neighborhood characteristics (numbers of dentists, grocery stores, and churches) were included in the regression models as continuous variables.

\section{Training and quality of social and behavioral data}

Written data collection protocols were developed and modified before the data collection phase. All examiners and interviewers were trained to administer their assigned questionnaires. All questionnaires were pretested before the start of the study and all questionnaires went through development and evaluation phases that included cognitive interviews with volunteers from the targeted community. During the study, interviewers were video or audiotaped and the tapes were reviewed by research coordinators or investigators to evaluate the adherence to protocols. The findings of the reviews were shared with the interviewers.

For logistical reasons related to the complexity of re-scheduling families for repeat examinations and time constraints, we have focused our efforts on assessing the reliability of ICDAS. The reliability of the Patient Hygiene Performance index was documented to be high in another study (19). The test-retest reliability of the exposure variables is not usually assessed because most measure attitudes and opinions and these may change over time. Like all studies in the field of social and behavioral sciences, this study suffers from recall and other biases associated with observational studies.

\section{Imputation}

Missing values in the variables used in this analysis were imputed using the IVEware software (21), a SAS-callable software application used to impute missing and nonsubstantive ('don't know' or 'refused) responses. IVEware performs multiple imputations using a procedure in which a sequence of regression models are fit and values are drawn from the predictive distributions. Missing values for dental caries, hygiene scores, neighborhoodlevel variables, age, and gender were not imputed as there were no missing data for the dental variables and the neighborhood variables. Educational status and sugar intake were not imputed as they were collected using instruments other than the questionnaire. For the other variables, the number of imputed cases ranged between one for the variables labeled employment status and reason for last visit to 57 for income status.

\section{Statistical analysis}

All interview and examination data were entered into a custom-written data entry program (written in Microsoft Access). The data were entered in duplicate by two different research staff. The two versions were checked for data entry errors and differences were reconciled by a third research staff. Data were analyzed using SAS (Version 9) and SUDAAN (Version 9.0.1). Weights were developed to adjust for differential nonresponse as well as unequal selection probabilities. Codes were created to allow computation of variances and test statistics that would account for stratification and cluster sample selection. The selected blocks were divided into 21 neighborhoods based upon demographic measures and poverty status. These neighborhood clusters were created for the purpose of extracting data from databases on community services. The intraclass correlation coefficients for the dental outcome variables was $<0.01$ indicating that there was no measurable clustering of dental caries within the neighborhoods.

Weighted bivariate estimates were computed using SUDAAN (Version 9.0.1) which accounted for clustering effects because of the sample design. ANOva and Tukey's Studentized range test were used to compare the mean values of the outcome variables by risk indicators. As the dependent variables represented counts of surfaces with significant dispersion (the variance of the count was 
larger than the mean), the negative binomial regression model was estimated using IVEware. Sampling weights and clustering effects caused by sampling were adjusted for using IVERware when the negative bionomial coefficients were computed. The negative binomial regression coefficients should be interpreted like logistic regression coefficients and can be exponentially transformed to provide an 'incidence ratio' or 'relative ratio' of disease rates in the exposed versus the reference groups. As a cross-sectional analysis our interest was not in evaluating the relative importance of the risk indicators of dental caries; hence, we did not transform the coefficients. When the longitudinal data were analyzed, a more relevant analysis of risk factors and their relative contribution to caries increments will be conducted. At that stage, relative ratios will be computed.

The negative binomial regression was chosen for this analysis because it is an appropriate model for analysis of 'over-dispersed' counts such as the number of noncavitated, cavitated, filled, or missing tooth surfaces [see chapter 8 in Ref. (22)].

\section{Results}

Table 2 presents the demographic, income, and educational status of the caregivers. The average age of the caregivers was 29.3 years. The majority $(46.2 \%)$ of the caregivers were between 25 and 34 years old. Over $95 \%$ of the caregivers were female and over half were unemployed at the time of the examination. About $40 \%$ of the caregivers lived in households that made less than $\$ 10,000$ per year: about $70 \%$ made less than $\$ 20,000$ per year. About $45 \%$ of the caregivers $(45.5 \%)$ did not finish high school.

Dental caries affected almost all the caregivers. Overall, $99.2 \%$ of the caregivers had at least one noncavitated carious lesion (ICDAS codes 1 and 2: see Table 3). Eighty-two percent of the caregivers had at least one tooth surface with a lesion classified at the ICDAS codes 3 to 6 (cavitated/ dentinal) stages. For secondary caries the overall prevalence is lower $(47.3 \%)$. The prevalence of restorations or sealants with caries ranged from $31.5 \%$ in 14 - to 24 -year-old caregivers to $52.9 \%$ in those $\geq 45$ years. The prevalence of caregivers with restorations or sealants with dentinal lesions or cavitated lesions (ICDAS codes 3-6) ranged from $17.2 \%$ in 14 - to 24 -year olds to $33.8 \%$ in caregivers aged $\geq 45$ years.
Table 2. Frequency distribution of adult dentate caregivers by selected demographic and employment characteristics

\begin{tabular}{|c|c|c|}
\hline Variable & Frequency & $\begin{array}{l}\text { Weighted } \\
\text { percentage }\end{array}$ \\
\hline \multicolumn{3}{|l|}{ Age (years) } \\
\hline $14-24$ & 342 & 34.0 \\
\hline $25-34$ & 464 & 46.2 \\
\hline $35-44$ & 143 & 14.2 \\
\hline$\geq 45$ & 56 & 5.6 \\
\hline \multicolumn{3}{|l|}{ Sex } \\
\hline Male & 55 & 4.6 \\
\hline Female & 950 & 95.5 \\
\hline \multicolumn{3}{|l|}{ Employment } \\
\hline Yes & 473 & 47.1 \\
\hline No & 532 & 52.9 \\
\hline \multicolumn{3}{|l|}{ Family's income } \\
\hline$<\$ 10000$ & 440 & 43.8 \\
\hline$\$ 10$ 000-\$19 999 & 278 & 27.7 \\
\hline$\$ 20000-\$ 29999$ & 164 & 16.3 \\
\hline$\geq \$ 30000$ & 123 & 12.2 \\
\hline \multicolumn{3}{|l|}{ Education level } \\
\hline Less than High School & 453 & 45.5 \\
\hline High School Graduate & 320 & 32.2 \\
\hline Some college or higher & 222 & 22.3 \\
\hline $\begin{array}{l}\text { Total sample size } \\
\text { (dentate only) }\end{array}$ & 1005 & \\
\hline $\begin{array}{l}\text { Total adults who were } \\
\text { edentulous }\end{array}$ & 16 & \\
\hline $\begin{array}{l}\text { Total adults (dentate and } \\
\text { edentulous) }\end{array}$ & 1021 & \\
\hline
\end{tabular}

The most common ICDAS-type carious lesions detected in all age groups were those classified with 'distinct visual change in enamel' (ICDAS code 2: see Table 4). The average number of these lesions range from 12.1 in caregivers aged 1424 years to 6.9 in those who were $\geq 45$ years. There were significant differences between age groups 14-24 and 44-45 years, and 14-24 and 45+ years.

The next most prevalent carious lesions at the ages of 14 to 24 and 25-34 years were those classified as ICDAS code 1 (first visible change in enamel). In caregivers aged 14-24 years, there were 9.5 tooth surfaces with these lesions. The average number of ICDAS 1 lesions declined to 7.5, 5.3 and then 3.0 by the age groups 25-34, 35-44, and $45+$ years, respectively. The mean number of ICDAS 1 lesions decreased significantly with age. The mean number of tooth surfaces with lesions classified with ICDAS codes 3,4, and 5, were lower than the mean of tooth surfaces classified with codes 1, 2, and 6 . The least prevalent status was ICDAS code 4 .

By the age of 35-44 years, extensive cavitated carious lesions (ICDAS code 6) were the second most common carious lesions in the adults. 
Table 3. Percentage (and standard error) of adults with at least one tooth surface with coronal caries by type and severity of primary or secondary carious lesions

\begin{tabular}{llllll}
\hline Dental caries measure & $14-24$ years & 25-34 years & 35-44 years & $\geq 45$ years & All age groups \\
\hline$n$ & 342 & 464 & 143 & 56 & 1005 \\
Primary caries (codes 1-2) & $99.7 \pm 0.2$ & $99.7 \pm 0.2$ & $96.9 \pm 1.9$ & $96.6 \pm 2.5$ & $99.2 \pm 0.3$ \\
Primary caries (codes 3-6) & $79.0 \pm 2.6$ & $83.3 \pm 1.7$ & $88.4 \pm 2.6$ & $73.2 \pm 5.3$ & $82.0 \pm 1.2$ \\
Secondary caries (codes 1-2) & $31.5 \pm 2.8$ & $56.3 \pm 3.1$ & $53.3 \pm 5.3$ & $52.9 \pm 5.9$ & $47.3 \pm 2.2$ \\
Secondary caries (codes 3-6) & $17.2 \pm 2.3$ & $33.2 \pm 3.5$ & $43.7 \pm 3.7$ & $33.8 \pm 5.2$ & $29.1 \pm 2.2$ \\
\hline
\end{tabular}

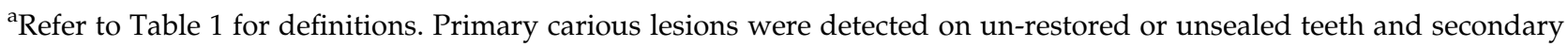
carious lesions are associated with restorations or sealants.

Table 4. Mean (and standard error) number of tooth surfaces by dental caries or restoration status

\begin{tabular}{|c|c|c|c|c|}
\hline Tooth status & $14-24$ years & 25-34 years & 35-44 years & $45+$ years \\
\hline Number of adults & 342 & 464 & 143 & 56 \\
\hline Sound & $136.7 \pm 1.3$ & $126.8 \pm 1.5$ & $103.2 \pm 3.3$ & $84.0 \pm 4.8$ \\
\hline First visible change in enamel (ICDAS 1) & $9.5 \pm 0.4$ & $7.5 \pm 0.3$ & $5.3 \pm 0.4$ & $3.0 \pm 0.8$ \\
\hline Distinct visual change in enamel (ICDAS 2) & $12.1 \pm 0.7$ & $11.5 \pm 0.4$ & $9.7 \pm 0.5$ & $6.9 \pm 1.0$ \\
\hline $\begin{array}{l}\text { Initial breakdown in enamel due to caries } \\
\text { with no visible dentin (ICDAS 3) }\end{array}$ & $2.2 \pm 0.2$ & $2.2 \pm 0.1$ & $1.9 \pm 0.2$ & $1.6 \pm 0.3$ \\
\hline $\begin{array}{l}\text { Noncavitated surface with underlying dark } \\
\text { shadow from dentin (ICDAS 4) }\end{array}$ & $0.6 \pm 0.1$ & $1.0 \pm 0.1$ & $1.0 \pm 0.2$ & $1.0 \pm 0.3$ \\
\hline Distinct cavity with visible dentin (ICDAS 5) & $0.9 \pm 0.1$ & $1.3 \pm 0.2$ & $1.3 \pm 0.2$ & $1.4 \pm 0.5$ \\
\hline Extensive distinct cavity with visible dentin (ICDAS 6) & $2.7 \pm 0.3$ & $5.1 \pm 0.5$ & $7.7 \pm 1.6$ & $6.6 \pm 1.5$ \\
\hline Amalgam restoration with no CARS ${ }^{\mathrm{a}}$ & $2.0 \pm 0.2$ & $3.9 \pm 0.3$ & $4.4 \pm 0.4$ & $4.5 \pm 0.8$ \\
\hline Amalgam restoration with early carious lesions ${ }^{b}$ & $0.8 \pm 0.1$ & $1.9 \pm 0.2$ & $1.7 \pm 0.2$ & $1.6 \pm 0.6$ \\
\hline Amalgam with caries in dentin & $0.2 \pm 0.03$ & $0.4 \pm 0.1$ & $0.7 \pm 0.1$ & $0.5 \pm 0.2$ \\
\hline Sealants with no CARS ${ }^{a}$ & $0.01 \pm 0.01$ & $0.01 \pm 0.01$ & $0.0 \pm 0.0$ & $0.02 \pm 0.02$ \\
\hline Crowns with no CARS & $0.05 \pm 0.04$ & $0.5 \pm 0.2$ & $0.8 \pm 0.3$ & $2.1 \pm 0.7$ \\
\hline $\begin{array}{l}\text { Other restorations (tooth colored or stainless steel) } \\
\text { with no CARS }\end{array}$ & $0.3 \pm 0.1$ & $0.4 \pm 0.1$ & $0.9 \pm 0.2$ & $0.8 \pm 0.3$ \\
\hline Missing teeth due to caries & $0.5 \pm 0.1$ & $1.8 \pm 0.2$ & $5.2 \pm 0.5$ & $8.0 \pm 1.0$ \\
\hline Missing teeth due to other reasons & $0.2 \pm 0.04$ & $0.6 \pm 0.1$ & $1.5 \pm 0.5$ & $2.8 \pm 0.8$ \\
\hline
\end{tabular}

${ }^{\mathrm{a}} \mathrm{CARS}$, caries adjacent to restorations or sealants.

${ }^{\mathrm{b}}$ Early carious lesions include ICDAS codes 1 or 2; caries in dentin includes ICDAS codes 3 to 6.

However, the mean number of these lesions per individual significantly declined after the age of 44 years, because of the significant increase in the number of missing teeth. The mean numbers of these lesions were significantly different between age groups 14-24 years and all older age groups as well as between age groups $25-34$ and 35-44 years.

The mean number of amalgam-filled tooth surfaces significantly increased between the age of 1424 years and the older age groups. However, after the age of 34 years, the mean number of amalgamfilled tooth surfaces with no carious lesions adjacent to the restoration was not statistically different from the mean detected in 25- to 34-year-old adults.

The mean number of missing teeth was significantly associated with age. By the age of 3544 years, adults lost 5.2 teeth on average because of dental caries. Adults $\geq 45$ years had lost a mean of 8.0 teeth because of dental caries. The mean number of teeth extracted for reasons other than dental caries more than doubled between the age groups of 35-44 and 45+ years, from 1.5 to 2.8.

Table 5 presents the mean number of tooth surfaces by status of dental caries (early or cavitated/dentinal) or filled for pits and fissures, mesialdistal surfaces, and buccal-lingual surfaces. Pits and fissures were the tooth sites with the highest caries experience. However, most of the carious lesions in pits and fissures were of the early or noncavitated type (ICDAS codes 1 and 2). On average, there were 15.6 pit-and-fissure surfaces with early carious lesions in 14- to 24-year olds. The average of these lesions per adult decreased significantly with age. By the age of 45 years and older the average number of lesions decreased to 7.5 per adult.

The mean number of cavitated/dentinal carious pit-and-fissure surfaces ranged from 4.1 in 14- to 24-year olds to a maximum of 5.2 in 35- to 44-year olds. This increase was not statistically significant. 
Dental caries in low-income African-American adults

Table 5. Mean (and standard error) number of decayed, missing and filled surfaces by type of tooth surface

\begin{tabular}{|c|c|c|c|c|c|}
\hline Type of tooth surface & 14-24 years & 25-34 years & 35-44 years & $45+$ years & Total \\
\hline \multicolumn{6}{|l|}{ Pits and fissures (54) } \\
\hline $\mathrm{D} 1^{\mathrm{a}}$ & $15.6 \pm 0.5$ & $14.6 \pm 0.4$ & $11.1 \pm 0.6$ & $7.5 \pm 1.0$ & $14.1 \pm 0.3$ \\
\hline $\mathrm{D} 2^{\mathrm{b}}$ & $4.1 \pm 0.3$ & $4.8 \pm 0.3$ & $5.2 \pm 0.6$ & $3.9 \pm 0.8$ & $4.6 \pm 0.2$ \\
\hline Filled $^{\mathrm{c}}$ & $1.9 \pm 0.2$ & $3.7 \pm 0.3$ & $4.2 \pm 0.4$ & $4.2 \pm 0.7$ & $3.2 \pm 0.2$ \\
\hline \multicolumn{6}{|c|}{ Buccal-lingual smooth tooth surfaces (64) } \\
\hline $\mathrm{D} 1^{\mathrm{a}}$ & $6.2 \pm 0.5$ & $4.8 \pm 0.2$ & $3.5 \pm 0.2$ & $1.7 \pm 0.3$ & $4.9 \pm 0.2$ \\
\hline $\mathrm{D} 2^{\mathrm{b}}$ & $1.4 \pm 0.1$ & $2.6 \pm 0.3$ & $3.5 \pm 0.6$ & $3.3 \pm 0.8$ & $2.3 \pm 0.2$ \\
\hline Filled $^{\mathrm{c}}$ & $0.2 \pm 0.04$ & $0.4 \pm 0.1$ & $0.9 \pm 0.2$ & $1.3 \pm 0.3$ & $0.4 \pm 0.1$ \\
\hline \multicolumn{6}{|c|}{ Mesial-distal smooth tooth surfaces (64) } \\
\hline $\mathrm{D} 1^{\mathrm{a}}$ & $0.9 \pm 0.1$ & $1.7 \pm 0.1$ & $2.6 \pm 0.2$ & $3.0 \pm 0.6$ & $1.6 \pm 0.1$ \\
\hline $\mathrm{D} 2^{\mathrm{b}}$ & $1.4 \pm 0.2$ & $3.1 \pm 0.3$ & $4.3 \pm 0.6$ & $4.3 \pm 0.8$ & $2.7 \pm 0.2$ \\
\hline Filled $^{\mathrm{c}}$ & $0.3 \pm 0.1$ & $0.8 \pm 0.1$ & $1.3 \pm 0.2$ & $2.3 \pm 0.5$ & $0.8 \pm 0.1$ \\
\hline \multicolumn{6}{|l|}{ All surfaces (182) } \\
\hline $\mathrm{D} 1^{\mathrm{a}}$ & $22.5 \pm 1.0$ & $21.1 \pm 0.5$ & $17.2 \pm 0.6$ & $12.1 \pm 1.2$ & $20.6 \pm 0.4$ \\
\hline $\mathrm{D} 2^{\mathrm{b}}$ & $7.0 \pm 0.5$ & $10.5 \pm 0.8$ & $13.0 \pm 1.8$ & $11.6 \pm 2.3$ & $9.6 \pm 0.5$ \\
\hline Filled $^{\mathrm{c}}$ & $2.4 \pm 0.2$ & $5.0 \pm 0.5$ & $6.3 \pm 0.6$ & $7.8 \pm 1.3$ & $4.4 \pm 0.3$ \\
\hline Missing tooth surfaces ${ }^{\mathrm{d}}$ & $3.1 \pm 0.6$ & $11.0 \pm 1.1$ & $32.2 \pm 2.7$ & $48.3 \pm 5.9$ & $12.8 \pm 0.9$ \\
\hline Missing teeth ${ }^{\mathrm{d}}$ & $0.5 \pm 0.1$ & $1.8 \pm 0.2$ & $5.2 \pm 0.5$ & $8.0 \pm 1.0$ & $2.1 \pm 0.2$ \\
\hline
\end{tabular}

${ }^{\mathrm{a}} \mathrm{D} 1$, noncavitated carious lesions (primary or secondary at ICDAS 1-2 level).

${ }^{b}$ D2, cavitated/dentinal carious lesions (primary and secondary at ICDAS 3-6 level).

${ }^{c}$ Filled sound.

${ }^{\mathrm{d}}$ Missing due to caries.

Filled tooth surfaces increased significantly in pits and fissures between the age groups 14-24 years and all older age groups but did not increase significantly between age groups after the age of 24 years.

The second most affected tooth surfaces were the buccal-lingual surfaces. On average in caregivers aged 14-24 years, there were 6.2 noncavitated lesions on smooth areas of the buccal and lingual surfaces. The mean number of noncavitated lesions on these surfaces for the age group 14-24 years was significantly different than the mean for the older age groups. After the age of 24 years, the mean values between age groups were not significantly different. The mean number of cavitated/dentinal lesions on buccal and lingual surfaces increased significantly between the age group of 14-24 years, and the age groups of 25-34 and 35-44 years.

On the mesial-distal surfaces, contrary to the other tooth surfaces, there were a higher mean number of visually detected cavitated/dentinal tooth surfaces than noncavitated tooth surfaces. There were significant increases in noncavitated and cavitated/dentinal surfaces among all age groups except between the age groups 35-44 and $45+$ years (Table 5). The mean number of filled mesial-and-distal tooth surfaces increased significantly with age.

Table 6 presents the findings of multivariate regression models (negative binomial) for five dental outcomes. We have included a new measure [total decayed tooth surfaces (DT)] in this analysis for comparison. The analysis of the risk indicators of the DT scores was presented in a previous paper (9).

Age was a consistent and significant indicator associated with all the dental outcome measures, except for the total number of decayed tooth surfaces (DT). The reason for this finding is that when the different stages of the carious process were measured, the differences in mean number of all decayed teeth among the age groups almost disappeared. For example, adults in the age group 25-34 years had a total of 30.1 decayed teeth (DT) and those in the age group 14-24 years had a mean of 29.6 DT.

Those who visited dentists for preventive care had a significantly lower mean number of cavitated/dentinal tooth surfaces, while those who had a recent dental visit had a significantly higher mean number of cavitated tooth surfaces. Caregivers with some college or higher had a significantly lower mean number of cavitated tooth surfaces than those with less than high school education. Excellent, good, or fair self-rating of oral health status was significantly associated with a lower mean number of cavitated tooth surfaces and missing teeth compared with poor rating. Caregivers who reported having a great deal or some pain also had a significantly higher mean number of noncavitated tooth surfaces than those who reported no pain at all. Those who worried a great deal 
Ismail et al.

Table 6. Multivariate regression model (negative binomial) coefficients and standard errors for four dental outcomes [noncavitated (D1), cavitated (D2), total decayed (DT), or filled tooth surfaces, and missing teeth] and individual and community risk indicators

\begin{tabular}{|c|c|c|c|c|c|c|c|c|c|c|}
\hline \multirow[b]{2}{*}{ Risk indicators } & \multicolumn{8}{|c|}{ Tooth surfaces } & \multicolumn{2}{|l|}{ Teeth } \\
\hline & D1 & SE & D2 & SE & DT & SE & Filled & SE & Missing & SE \\
\hline \multicolumn{11}{|l|}{ Demographics } \\
\hline \multicolumn{11}{|l|}{ Gender } \\
\hline Female & -0.03 & 0.11 & 0.34 & 0.25 & 0.08 & 0.10 & -0.17 & 0.31 & $-0.61^{*}$ & 0.23 \\
\hline Male & Ref & & Ref & & Ref & & Ref & & Ref & \\
\hline \multicolumn{11}{|l|}{ Age } \\
\hline $14-24$ years & Ref & & Ref & & Ref & & Ref & & Ref & \\
\hline $25-34$ years & -0.04 & 0.05 & $0.30^{* * *}$ & 0.08 & 0.04 & 0.04 & $0.57^{* * *}$ & 0.16 & $1.07^{* * *}$ & 0.17 \\
\hline $35-44$ years & $-0.24^{* * *}$ & 0.07 & $0.49^{* *}$ & 0.16 & 0.00 & 0.06 & $0.89^{* * * *}$ & 0.17 & $2.38^{* * *}$ & 0.21 \\
\hline 45 and older & $-0.55^{* * *}$ & 0.11 & $0.49^{*}$ & 0.23 & -0.21 & 0.13 & $0.94^{* * *}$ & 0.18 & $3.32^{* * *}$ & 0.24 \\
\hline \multicolumn{11}{|l|}{ Employment } \\
\hline Yes & 0.01 & 0.04 & -0.04 & 0.07 & -0.02 & 0.03 & 0.05 & 0.13 & 0.12 & 0.10 \\
\hline No & Ref & Ref & Ref & Ref & Ref & & & & & \\
\hline \multicolumn{11}{|l|}{ Family's income } \\
\hline Less than $\$ 10000$ & Ref & & Ref & & Ref & & Ref & & Ref & \\
\hline Between $\$ 10000$ and 20000 & 0.05 & 0.04 & -0.01 & 0.12 & 0.04 & 0.05 & 0.10 & 0.17 & 0.14 & 0.14 \\
\hline Between $\$ 20000$ and 30000 & -0.07 & 0.06 & -0.05 & 0.13 & -0.03 & 0.06 & 0.28 & 0.19 & 0.14 & 0.22 \\
\hline More than $\$ 30000$ & 0.11 & 0.07 & 0.00 & 0.24 & 0.09 & 0.09 & 0.27 & 0.20 & -0.13 & 0.17 \\
\hline \multicolumn{11}{|l|}{ Education Level } \\
\hline Less than High School & Ref & Ref & Ref & Ref & Ref & & & & & \\
\hline High School Graduate & -0.06 & 0.05 & -0.04 & 0.10 & -0.05 & 0.04 & 0.23 & 0.15 & 0.01 & 0.11 \\
\hline Some college or higher & 0.04 & 0.05 & $-0.20^{*}$ & 0.10 & -0.01 & 0.04 & 0.27 & 0.18 & -0.11 & 0.23 \\
\hline \multicolumn{11}{|l|}{ Access to care } \\
\hline \multicolumn{11}{|l|}{ Type of insurance } \\
\hline Medicaid & -0.07 & 0.08 & -0.14 & 0.17 & -0.08 & 0.09 & 0.04 & 0.22 & -0.25 & 0.15 \\
\hline Private & 0.06 & 0.05 & 0.10 & 0.10 & 0.07 & 0.04 & -0.02 & 0.17 & 0.13 & 0.14 \\
\hline None & Ref & & Ref & & Ref & & Ref & & Ref & \\
\hline \multicolumn{11}{|l|}{ Last visit to dentist } \\
\hline$<2$ years ago & -0.19 & 0.15 & $0.85^{*}$ & 0.38 & 0.18 & 0.16 & 0.81 & 0.44 & 0.40 & 0.65 \\
\hline $2-5$ years ago & -0.13 & 0.12 & $0.75^{*}$ & 0.38 & 0.22 & 0.17 & 0.40 & 0.46 & -0.19 & 0.62 \\
\hline$>5$ years ago or never & Ref & & Ref & & Ref & & Ref & & Ref & \\
\hline \multicolumn{11}{|l|}{ Reason for visit } \\
\hline Preventive & 0.14 & 0.14 & $-0.95^{*}$ & 0.38 & -0.25 & 0.16 & -0.18 & 0.42 & -0.03 & 0.63 \\
\hline Both & 0.16 & 0.12 & -0.74 & 0.40 & -0.20 & 0.17 & 0.12 & 0.45 & 0.64 & 0.59 \\
\hline Treatment & 0.20 & 0.13 & -0.41 & 0.39 & -0.07 & 0.16 & 0.02 & 0.49 & 1.08 & 0.62 \\
\hline Never been to dentist & Ref & & Ref & & Ref & & Ref & & Ref & \\
\hline \multicolumn{11}{|l|}{ Quality of life } \\
\hline Rating of oral health & & & & & & & & & & \\
\hline Excellent/very good & 0.16 & 0.09 & $-1.11^{* * *}$ & 0.26 & -0.14 & 0.08 & -0.35 & 0.27 & $-0.98^{* * *}$ & 0.24 \\
\hline Good & 0.09 & 0.07 & $-0.82^{* * *}$ & 0.19 & $-0.18^{* *}$ & 0.07 & -0.19 & 0.21 & $-0.85^{\mathrm{ad}}$ & 0.16 \\
\hline Fair & $0.12^{*}$ & 0.06 & $-0.38^{\mathrm{ad}}$ & 0.10 & -0.07 & 0.05 & 0.04 & 0.16 & -0.07 & 0.13 \\
\hline Poor & Ref & & Ref & & Ref & & Ref & & Ref & \\
\hline Pain caused by teeth & & & & & & & & & & \\
\hline A great deal & $0.18^{*}$ & 0.07 & 0.22 & 0.16 & $0.19^{* * *}$ & 0.07 & -0.27 & 0.26 & -0.00 & 0.24 \\
\hline Some pain & $0.12^{*}$ & 0.05 & 0.03 & 0.18 & 0.07 & 0.06 & -0.07 & 0.19 & -0.21 & 0.16 \\
\hline A little pain & 0.03 & 0.05 & 0.09 & 0.15 & 0.06 & 0.06 & -0.16 & 0.24 & -0.05 & 0.15 \\
\hline No pain at all & Ref & & Ref & & Ref & & Ref & & Ref & \\
\hline Worried about teeth & & & & & & & & & & \\
\hline A great deal & 0.01 & 0.06 & $0.31^{*}$ & 0.15 & 0.10 & 0.06 & $0.58^{*}$ & 0.23 & 0.24 & 0.15 \\
\hline Somewhat & -0.03 & 0.07 & 0.05 & 0.12 & -0.01 & 0.05 & 0.40 & 0.22 & 0.26 & 0.16 \\
\hline A little & 0.05 & 0.06 & 0.10 & 0.17 & 0.05 & 0.07 & 0.36 & 0.21 & 0.21 & 0.16 \\
\hline Not at all & Ref & & Ref & & Ref & & Ref & & Ref & \\
\hline Behaviors & & & & & & & & & & \\
\hline Hygiene Performance Index & 0.01 & 0.07 & $0.81^{* * *}$ & 0.15 & $0.30^{* * *}$ & 0.07 & -0.35 & 0.23 & 0.04 & 0.22 \\
\hline Total sugar intake $(\mathrm{g})$ & 0.00 & 0.00 & 0.00 & 0.00 & 0.00 & 0.00 & $-0.00^{*}$ & 0.00 & 0.04 & 0.05 \\
\hline Neighborhood characteristics - Cet & asus 2000 & & & & & & & & & \\
\hline SES score (continuous) & -0.01 & 0.01 & -0.00 & 0.03 & -0.01 & 0.01 & 0.01 & 0.05 & 0.07 & 0.06 \\
\hline No. dentists (continuous) & 0.00 & 0.01 & $-0.05^{*}$ & 0.02 & -0.01 & 0.01 & -0.03 & 0.05 & -0.01 & 0.03 \\
\hline
\end{tabular}


Table 6. Continued

\begin{tabular}{|c|c|c|c|c|c|c|c|c|c|c|}
\hline \multirow[b]{2}{*}{ Risk indicators } & \multicolumn{8}{|c|}{ Tooth surfaces } & \multicolumn{2}{|l|}{ Teeth } \\
\hline & D1 & SE & $\mathrm{D} 2$ & SE & DT & SE & Filled & SE & Missing & SE \\
\hline No. grocery stores (continuous) & 0.01 & 0.01 & $0.04^{*}$ & 0.02 & $0.02 *$ & 0.01 & -0.00 & 0.03 & -0.04 & 0.03 \\
\hline No. churches (continuous) & $-0.01^{*}$ & 0.00 & -0.01 & 0.01 & $-0.01^{*}$ & 0.00 & -0.00 & 0.01 & $0.02^{*}$ & 0.01 \\
\hline Dispersion & $0.17^{* * *}$ & & $1.05^{* * *}$ & & $0.20^{* * *}$ & & $2.04^{* * *}$ & & $1.35^{* * *}$ & \\
\hline
\end{tabular}

${ }^{a} \mathrm{D} 1$, noncavitated carious lesions (ICDAS codes 1 and 2); D2, cavitated carious lesions (ICDAS codes 3-6); DT, total number of decayed tooth surfaces (ICDAS codes 1-6).

${ }^{*} P<0.05 ;{ }^{* *} P<0.01 ;{ }^{* * *} P<0.001$.

had significantly higher mean numbers of cavitated lesions and filled tooth surfaces than those who did not at all worry about their teeth. Oral hygiene status was significantly associated with a higher number of cavitated lesions.

The number of dentists in a neighborhood, an indicator of income status in a community, was negatively associated with the number of cavitated lesions. The total sugar intake in grams was negatively associated with the number of filled tooth surfaces. The number of churches was negatively associated with noncavitated carious lesions and the number of grocery stores was positively associated with the number of cavitated tooth surfaces. The number of churches was positively associated with the number of missing teeth.

\section{Discussion}

This epidemiological study is a cross-sectional investigation of the community and individual determinants of dental caries. The findings are limited by the cross-sectional nature of the data and the potential biases associated with interview data. Another limitation is related to the quality and completeness of the neighborhood information extracted from the online yellow pages directory and used to obtain the number of dentists, grocery stores, and churches. Hence, some underestimation in these exposures is possible. These errors may bias the point estimates towards the null value, under-estimating the true relationship. An advantage of this study is the cohort design. We have collected 2-year follow-up data from the caregivers and their children and future analyses will investigate causal relationships between dental caries and the risk indicators described in this study.

The study presents several new findings. First, this large study presents, for the first time, data on the stages of the carious process in a high-risk population in the United States. Secondly, the models estimated in the study incorporated community and individual risk indicators. Thirdly, the analysis focused on the components of the carious process, which, as this study has found, have different determinants.

Noncavitated, cavitated, filled, and missing components of the carious process have different distributions and determinants. The increased in the mean number of missing teeth after the age of 34 years may not reflect the increased burden of dental caries with age, but rather the type of dental care available to this population. The finding that the number missing for reasons other than dental caries increased significantly at each age group (Table 4) also raises questions as to whether dental caries is the reason for the rapid increase in missing teeth. In the NHANES III, the mean number of missing teeth in African-American adults was 6.8, which represented $55 \%$ of the total number of decayed, missing, and filled teeth. African-American adults in 1999-2002 had the highest percentage of missing teeth compared with white-Americans (30.4\%) and Mexican-Americans (41.4\%) (5). Another explanation for the high numbers of missing teeth may be the low quality of dental insurance coverage or the lack of dental insurance in this population. There is also evidence from a previous study in the same geographic area that some African-Americans, more often than white-Americans, tend to prefer extraction of badly decayed teeth to restoring them (23).

The use of ICDAS provided important information on caries distribution. Early noncavitated carious lesions were the most common type of lesions found in this population. These lesions were mostly located in pits and fissures. This finding is similar to those reported for children by Ismail et al. (13). Data on the prevalence of noncavitated carious lesions in African-American adults are not available. The risk indicators associated with noncavitated carious lesions were age, education status, reporting of a history of a great 
deal of pain caused by teeth and the number of churches.

Tellez et al. (9) has previously reported on the negative association between the number of churches in a community and the mean number of total decayed tooth surfaces. This analysis shows that the association was only found with the number of noncavitated carious lesions. Tellez et al. (9) hypothesized that the number of churches may indicate micro-differences in income status in low-income communities. Churches in Detroit, like other communities, depend on the donation of the worshippers. One could speculate that if worshippers in an area have more expendable income, then the number of churches would increase in the area. Additionally, there is evidence that religiosity may be associated with health and coping with illness (24, 25). Organized religiosity had an indirect positive effect on the association between being 'black' and mental health (25). Religiosity also provides social support for African-American caregivers (26); and the presence of social support was found to be associated with healthy behaviors (27). A recent study on the association between chronic diseases and religious involvement by African-American women on the east side of Detroit (similar area to this study) found that women with religious behaviors attending church and active in their community church, had lower depressive symptoms and chronic diseases, such as asthma and arthritis, when compared with women who do not share the same beliefs or behaviors (28). These studies all point to a positive association between religious beliefs and social support provided by faith-based organizations on disease development. The question that will be evaluated in the analysis of the cohort data is why these factors influence the progression of caries. We expect that positive healthy behaviors associated with church-active individuals lead to lower initiation of dental caries, estimated by ICDAS codes 1 and 2, and consequently, to a lower mean number of cavitated carious lesions.

Patient oral hygiene performance was significantly and positively associated with the number of cavitated tooth surfaces. This association did not exist with noncavitated carious lesions. It is possible that those with improved oral hygiene status and more frequent exposure to fluoridated toothpaste were less likely to have their noncavitated lesions progress to cavitation compared with those with poor oral hygiene status. Fluoride acts in a similar way. The mean number of 'enamel' or noncavitated lesions were similar between fluoridated and nonfluoridated communities in the Netherlands; however, the progression from 'enamel' to cavitated lesions was significantly lower in the fluoridated community than in the nonfluoridated one (29). While oral hygiene status has been inconsistently associated with dental caries, a recent study found that a targeted and focused oral hygiene program in adolescents, as a major component of an overall preventive program, can significantly reduce dental caries (30). When the cohort analysis is conducted within a year, this finding will be explored in detail.

There was a positive association between the number of cavitated carious lesions and the number of grocery stores and a negative association with the number of dentists in a neighborhood. Grocery stores in low-income neighborhoods are small and do not provide healthy foods such as fruits, vegetables, whole-grain products, and lowfat meat (31). Observation indicates that these smaller grocery stores provide more sugar snacks and drinks in these low-income neighborhoods, which may explain this finding. The number of dentists reflects the income status of the community. Dental practices are located in areas where there are potential patients who can afford to pay for the cost of dental care.

A consistent finding in the study was the association between reports of 'a great deal' of worry about teeth and cavitated and filled tooth surfaces. The adults participating in this study could evaluate their oral health status and the need for dental care. There is no previous study of this association in African Americans. In elderly persons in Greece, Tsakos (32) reported an association between decayed teeth and impact on daily living. The predictive ability of self-reports of 'worry' or 'pain' related to the teeth will be investigated using the cohort data.

This study found that dental caries is highly prevalent in African-American adults. Noncavitated pits and fissures were the most prevalent caries stage. Oral hygiene status was significantly associated with the number of cavitated lesions. Missing teeth increased rapidly after the age of 34 years indicating the possibility that in this low-income minority population caries is managed through extraction or the people prefer to have their teeth extracted rather than restored. Individuals who reported that they visited dentists for preventive care had a significantly lower mean number of cavitated lesions than those who visited a dentist for dental treatment. 


\section{Acknowledgments}

This study was supported with funding from the National Institute on Dental and Craniofacial Research (NIDCR) grant no. U-54 DE 14261-01, the Delta Dental Fund of Michigan, and the University of Michigan's Office of Vice President for Research. The authors thank the staff of the project for their diligence and commitment. The following individuals contributed to this research: Keri Frisch, Charity Hicks, Laprisha Berry, Lindsey Larkin, Maureen Kreple, Nikia Thomas, and Danette Gray. The dentists who collected the data are Drs. James Betz, David LaCharite, Lorelei Claiborne, Gloria Smith, Hana Hasson, and Woosung Sohn.

\section{References}

1. Brown LJ, Wall TP, Lazar V. Trends in untreated caries in primary teeth of children 2 to 10 years old. J Am Dent Assoc 2000;1313:93-100.

2. Edelstein BL. Disparities in oral health and access to care: findings of national surveys. Ambul Pediatr 2002;2(Suppl. 2):141-7.

3. Vargas CM, Crall JJ, Schneider DA. Sociodemographic distribution of pediatric dental caries: NHANES III, 1988-1994. J Am Dent Assoc 1998;129:1229-38.

4. Barrow SL, Xionan X, LeGeros A, Mijares DQ, LeGros RZ, Galvis DL et al. Dental caries prevalence among a sample of African-American adults in New York City. Dent Clin North Am 2003;47:57-65.

5. Beltrán-Aguilar ED, Barker LK, Canto MT, Dye BA, Gooch BF, Griffin SO et al. Surveillance for Dental Caries, Dental Sealants, Tooth Retention, Edentulism, and Enamel Fluorosis United States, 1988-1994 and 1999-2002. MMWR 2005;54:1-44.

6. Radike AW. Criteria for diagnosing dental caries. In: Proceedings of the Conference on the Clinical Testing of Cariostatic Agents. Chicago, IL, USA: American Dental Association; 1968. p. 87-8.

7. International Caries Detection and Assessment System Coordinating Committee. Rationale and evidence for the International Caries Detection and Assessment System (ICDASII). In: Stookey G, editor. Proceedings of the 2005 Annual Indiana Conference, July 6-9, 2005. Indianapolis, IN: Indiana University School of Dentistry; 2006. in press.

8. Subramanian SV, Chen JT, Rehkopf DH, Waterman PD, Krieger N. Racial disparities in context: a multilevel analysis of neighborhood variations in poverty and excess mortality among black populations in Massachusetts. Am J Public Health 2005;95:260-5.

9. Tellez M, Sohn W, Burt BA, Ismail AI. Assessment of the relationship between neighborhood characteristics and dental caries severity among low-income African-Americans: a multilevel approach. J Public Health Dent 2006;66:30-6.

10. Ismail AI, Sohn W, Tellez M, Sen A, Amaya A. The International Caries Detection and Assessment System (ICDAS): an integrated system for measuring dental caries. Community Dent Oral Epidemiol 2006;34:1-9.

11. Ismail AI. Visual and visuo-tactile detection of dental caries. J Dent Res 2004;82(Spec Iss C):C56-C66.
12. Pitts NB, Fyffe HE. The effect of varying diagnostic thresholds upon clinical caries data for a low prevalence group. J Dent Res 1988;67:592-6.

13. Ismail AI, Brodeur JM, Gagnon P, Payette M, Picard D, Hamalian T et al. Prevalence of non-cavitated and cavitated carious lesions in a random sample of 7-9year-old schoolchildren in Montreal, Quebec. Community Dent Oral Epidemiol 1992;20:250-5.

14. Ekstrand KR, Ricketts DN, Kidd EA. Reproducibility and accuracy of three methods for assessment of demineralization depth of the occlusal surface: an in vitro examination. Caries Res 1997;31:224-31.

15. Fyffe HE, Deery C, Nugent ZJ, Nuttall NM, Pitts NB. Effect of diagnostic threshold on the validity and reliability of epidemiological caries diagnosis using the Dundee Selectable Threshold Method for caries diagnosis (DSTM). Community Dent Oral Epidemiol 2000;28:42-51.

16. Ricketts DNJ, Ekstrand KR, Kidd EAM, Larsen T. Relating visual and radiographic ranked scoring systems for occlusal caries detection to histological and microbiological evidence. Operative Dent 2002;27:231-7.

17. Block PL, Lobene RR, Derdivanis JP. A two-tone dye test for dental plaque. J Periodontol 1972;43:423-6.

18. Podshadley AG, Haley JV. A method for evaluating oral hygiene performance. Public Health Rep 1968;83:259-63.

19. Mander CI, Mainwaring PJ. Assessment of the validity of two plaque indices. Community Dent Oral Epidemiol 1980;8:139-41.

20. Silness J, Loe H. Periodontal disease in pregnancy. II. A correlation between oral hygiene and periodontal conditions. Acta Odontol Scand 1964;22:121-35.

21. Raghunathan TE, Lepkowski JM, VanHoewyk J, Solenberger P. A Multivariate technique for multiply imputing missing values using a sequence of regression models. Survey Methodol 2001;29:85-95. This article and information regarding access to IVEware is available on the web at http://www.isr.umich. edu/src/smp/ive.

22. Long J. Scott. Regression Models for Categorical and Limited Dependent Variables. Thousand Oaks, CA: Sage Publications; 1997.

23. Ismail AI, Birch S, Sohn W, Lepkowski JM, Belli RF. Utilities of dentin regeneration among insured and uninsured adults. Community Dent Oral Epidemiol 2004;32:55-66.

24. Holt C, Lewellyn LA, Rathweg MJ. Exploring religion-health mediators among African American parishioners. J Health Psychol 2005;10:511-27.

25. Franzini L, Ribble JC, Wingfield KA. Religion, sociodemographic and personal characteristics, and selfreported health in whites, blacks, and Hispanics living in low-socioeconomic status neighborhoods [see comment]. Ethn Dis 2005;15:469-84.

26. Carothers SS, Borkowski JG, Lefever JB, Whitman TL. Religiosity and the socioemotional adjustment of adolescent mothers and their children. J Family Psych 2005;19:263-75.

27. Black AR, Cook JL, Murry VM, Cutrona CE. Ties that bind: implications of social support for rural, partnered African American women's health functioning. Womens Health Issues 2005;15:216-23. 
Ismail et al.

28. van Olphen J, Schulz A, Israel B, Chatters L, Klem L, Parker E et al. Religious involvement, social support, and health among African-American women on the east side of Detroit. J Gen Intern Med 2003;18:549-57.

29. Groeneveld A. Longitudinal study of prevalence of enamel lesions in a fluoridated and non-fluoridated area. Community Dent Oral Epidemiol 1985;13:15963.

30. Ekstrand KR, Christiansen ME. Outcomes of a nonoperative caries treatment programme for children and adolescents. Caries Res 2005;39:455-67.
31. Nicklas TA, McQuarrie A, Fastnaught C, O'Neil CE. Efficiency of breakfast consumption patterns of ninth graders: nutrient-to-cost comparisons. J Am Diet Assoc 2002;102:226-33.

32. Tsakos G, Marcenes W, Sheiham A. The relationship between clinical dental status and oral impacts in an elderly population. Oral Health Prev Dent 2004;2:211-20. 